\title{
Le cadavre en substance : perte d'odeurs et principe vital
}

The corpse in subtance: loss of smells and vital principle

Joël Candau

\section{(2) OpenEdition}

1 Journals

Édition électronique

URL : https://journals.openedition.org/tc/6895

DOI : $10.4000 /$ tc. 6895

ISBN : 1952-420X

ISSN : 1952-420X

Éditeur

Éditions de l'EHESS

Édition imprimée

Date de publication : 19 juin 2013

Pagination : 110-125

ISBN : 978-2-7351-1637-9

ISSN : 0248-6016

\section{Référence électronique}

Joël Candau, «Le cadavre en substance : perte d'odeurs et principe vital », Techniques \& Culture [En ligne], 60 | 2013, mis en ligne le 19 juin 2016, consulté le 29 septembre 2022. URL : http:// journals.openedition.org/tc/6895; DOI : https://doi.org/10.4000/tc.6895 







\section{LE CADAVRE EN SUBSTANCE}

\section{Perte d'odeurs et principe vital}

Ce que nous appelons « cadavre » est la matière organique constitutive d'un individu décédé, telle qu'elle se donne à voir, à sentir ou à toucher, ou telle que nous l'imaginons quand le cadavre est absent ou disparu. Mon objectif, ici, est de considérer les effets cognitifs et culturels de cette matière, ce qui revient à explorer les pratiques et représentations induites par la substance même du cadavre ou, plus modestement, certaines d'entre elles compte tenu du caractère inévitablement limité de mes données. Après avoir balisé théoriquement mon analyse dans la première partie de l'article - en évoquant la théorie de la cognition incarnée (C.I.) et l'anthropologie des substances -, je rappelle dans la partie suivante que tout au long de son histoire évolutive notre espèce a entretenu une familiarité étroite avec le cadavre et sa transformation en charogne. Suivant la théorie de la C.I., je fais l'hypothèse que cette présence massive de la substance cadavérique dans la très longue durée a induit des représentations de premier degré (concrètes) qui ont elles-mêmes servi de supports à des représentations de second degré, plus abstraites. Je soutiens qu'une de ces représentations est celle de principe vital, la perte ou la transformation de la substance du cadavre étant perçue comme la dissipation de ce principe. Dans la troisième et dernière partie, je défends l'idée d'un lien entre la décomposition du corps, la perte d'odeurs et la dissipation du principe vital, thèse que j'étaye par des données ethnographiques recueillies dans la région de Nice. Je conclus par quelques considérations anthropologiques hautement spéculatives sur l'origine de la pensée dualiste.
"Toute chose représente la substance de sa perte" (James, H. Les Ambassadeurs, Paris: Le Bruit du temps, $2010: 471$ ). 
région du visage, le muscle élévateur de la lèvre supérieure ou quadratus labii superioris (Chapman et al. 2009). Par ailleurs, les mêmes régions du cerveau sont activées (Kross et al. 2011, Lieberman \& Eisenberger 2009) d'une part quand on éprouve un plaisir physique ou social (par exemple, après avoir été charitable), d'autre part quand on subit une douleur physique ou sociale (par exemple, être l'objet d'exclusion d'un groupe d'amis). Ce chevauchement entre deux registres a priori distincts (celui des expériences sensorielles et celui des jugements moraux) trouve en outre son expression en langue. Dans notre entourage, s'il nous arrive de trouver certaines personnes « exquises » ou « délicieuses », d'autres nous paraissent « répugnantes » ou " dégoûtantes », ce qui laisse à nouveau supposer que ces attributions morales subissent l'influence d'évaluations sensorielles, comme cela est d'ailleurs suggéré en français et dans de très nombreuses langues où on compte une grande variété d'expressions qui mettent en relation perceptions sensorielles et jugement des individus: « je ne peux pas le voir», « je ne peux pas le sentir », « il est puant », « il me sort par le nez », « il me sort par les yeux », « il me casse les oreilles », etc.

On sait, par ailleurs, que l'exposition d'individus à des odeurs évoquant des produits de nettoyage les conduit à être plus attentifs à la propreté de leur environnement lors d'une tâche liée à l'alimentation (Holland et al. 2005). Mais cette influence des sens chimiques sur les notions de propreté physique s'étend jusqu'à des notions plus abstraites, telles que la pureté morale. Des sujets conduits expérimentalement à adopter des comportements menaçant leur intégrité morale et qui doivent ensuite choisir entre des produits de nettoyage et d'autres objets choisissent bien plus souvent les produits de nettoyage que les individus appartenant au groupe de contrôle (Zhong \& Liljenquist 2006). D’autres expériences ont montré que des sujets chez qui le concept de propreté a été suscité grâce à des mots le dénotant, ou qui ont eu la possibilité de se laver après avoir eu une expérience de dégoût, portent des jugements moins sévères lorsqu'ils sont confrontés à des dilemmes moraux

Laver ses péchés






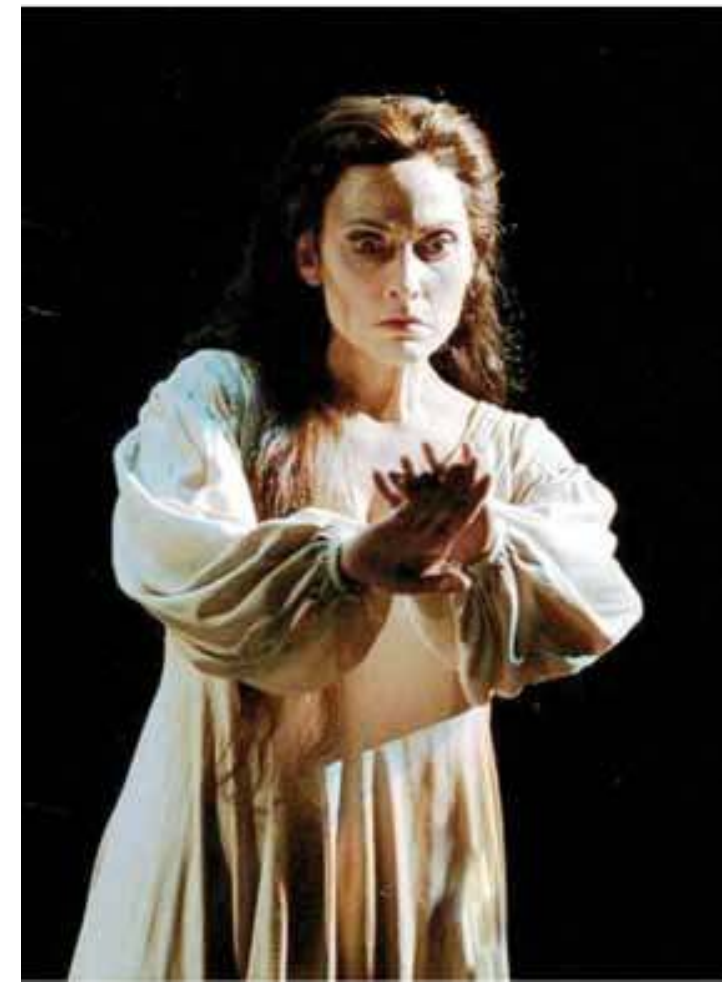

Macbeth

Dame Macbeth, somnambule, essayant de laver le sang de Duncan sur ses mains. "Out, damned spot! Out, I say!" s'écrie Lady Macbeth

(V, 1) dans la célèbre tragédie shakespearienne. que les individus d'un groupe de contrôle chez qui le concept n'a pas été activé ou qui n'ont pas pu se laver après l'expérience (Schnall et al. 2008). Selon Liljenquist et al. 2010, les odeurs des produits de nettoyage non seulement induisent des comportements hygiéniques mais aussi des comportements vertueux, en augmentant les inclinations à la confiance réciproque et à l'aide charitable. Inversement, les nuisances olfactives peuvent altérer les représentations qu'un groupe se fait d'un territoire ancestral, comme l'ont montré les travaux de Jackson (2011) dans une réserve aborigène de l'Ontario située au sein de la «Vallée Chimique » canadienne. Dans un registre proche, l'intensité de la crainte d'une contamination microbienne (Park et al. 2011) et la réalité de ce danger (van Leeuwen et al. 2012) semblent être de bons prédicteurs d'opinions ou d'attitudes politiques qualifiées de conservatrices telles que, par exemple, des préjugés à l'encontre des personnes obèses ou une préoccupation prononcée de pureté morale, d'autorité et de loyauté à l'endroit du groupe d'appartenance. Enfin, faut-il rappeler cette photo terrible prise à Vienne en mars 1938 où l'on voit des nazis autrichiens et des habitants en train d'observer avec une satisfaction évidente des Juifs forcés à nettoyer les trottoirs ${ }^{1}$ : le lien est ici troublant entre l'obsession hygiénique et le fantasme de "pureté » de la « race » ou de « purification » ethnique.

"Out, damned spot! Out, I say! " s'écrie Lady Macbeth (V, I) dans la célèbre tragédie shakespearienne. En faisant disparaître les taches de sang qu'elle a sur les mains, la future reine espère se laver de la souillure que représente le meurtre de Duncan. La propreté physique retrouvée lui permettrait de recouvrer la pureté morale. Cet « effet Macbeth », fondé sur l'idée qu'on peut littéralement « laver » ses péchés, est documenté dans de nombreuses cultures où il est courant que des pratiques religieuses soient précédées d'ablutions (Al-Ghousl, Al-Woudhou', mikveh, baptême, etc.). À la pureté du corps (absence de contamination, pollution ou souillure) répondrait la pureté morale. Dans mes travaux sur les odeurs, j'ai été frappé par le fait que les professionnels (fossoyeurs, employés de la morgue, médecins légistes, infirmières, sapeurs pompiers) évoluant dans des environnements sensoriels sévères insistaient tous sur l'importance de la douche après le travail. Cette pratique ne semble pas seulement motivée par un souci de propreté physique, si j'interprète correctement les propos d'infirmières qui, après s'être trouvées en présence d'une odeur d'infection intense, se douchent aussitôt leur travail achevé dans le but, disent-elles, « d'enlever l'intérieur et l'extérieur » (Candau 2000). Par ailleurs, les représentations moralement « clivantes » de l'odeur de l'Autre sont sous-jacentes dans le discours des professionnels qui y sont confrontés. Qu'il s'agisse de l'odeur du SDF, du malade ou de la personne âgée (vivante ou morte), elle participe à chaque fois d'une stigmatisation plus ou moins euphémisée de celui qui est essentialisé dans son altérité en grande partie parce qu'il sent autrement. Cette propriété est elle-même appréhendée de manière d'autant plus prononcée par le percevant que, par le biais d'autres sens (la vue principalement, le toucher parfois), l'individu perçu a été 
préalablement assigné dans la catégorie d'une altérité radicale (Candau 2001; Candau \& Jeanjean 2006). Tous ces phénomènes, qui sont sous l'emprise des affects (Candau 2010a) et restent inconnus de notre système délibératif, suggèrent ici encore qu'au fil de l'évolution le dégoût d'ordre moral aurait pris au sein de notre espèce la forme d'un prolongement et d'une extension du dégoût physique dont la finalité est de protéger le corps d'agressions biologiques telles que des germes, de la nourriture toxique, etc. (Haidt \&Joseph 2008; Moll et al. 2005).

On doit alors admettre que les stimuli sensoriels entrent en jeu dans l'élaboration de représentations abstraites, celles-ci étant enracinées dans des perceptions sensorielles de bas niveau. Ces perceptions s'appliquent à toute la matière qui nous entoure et qui est faite de substances diverses. Dans la perspective de la C.I., on peut supposer que ces substances jouent un rôle déterminant car, du fait de leur constance ontologique elles ne cessent d'être exposées à un traitement cognitif, la plupart du temps à notre insu. Je développe mon argument à partir du cadre conceptuel proposé par Hahn \& Soentgen (2010). Ils rappellent d'abord la distinction classique entre la chose, qui est de la matière formée à partir de substance(s) et qui est countable, et la substance, qui est de la matière sans forme et qui est généralement uncountable, distinction que l'on peut rapprocher de celle de Quine (1977) entre les count nouns et les mass terms. On n'évalue jamais la substance en nombre (how many) mais en quantité (how much). Hahn \& Soentgen défendent ensuite la thèse de la non-séparabilité des choses et de la séparabilité des substances. Si je sépare les différents composants physiques qui constituent l'ordinateur sur lequel je suis en train d'écrire, ces composants cesseront d'être la chose que nous appelons ordinateur. En revanche, la substance est arbitrairement divisible, en ce sens que n'importe quel produit de sa division continue à tomber sous le concept qui la désigne. Je peux séparer autant de fois que je veux l'eau que je viens de verser dans mon verre, ou le miel que j’ai mangé ce matin sur des tartines pour mon petit déjeuner. Toutes les parties que j'obtiendrai, aussi infimes soient-elles, conserveront leur ontologie ${ }^{2}$ qui est d'être de l'eau ou du miel et je continuerai à les dénommer ainsi. En termes kantiens, la substance est assimilable à ce qui demeure « au milieu de tous les changements dans le monde » (Kant 1980 : 921) ou, si l'on veut, la matière dont la quiddité reste inchangée nonobstant ses accidents.

Il y a toutes sortes de substances, mais certaines sont fondamentales pour les êtres humains parce qu'elles sont vitales: l'eau, le sang, le lait ou le sperme. À ce titre, elles ont généralement ce que Carsten (2011) appelle une forte « capacité métaphorique ». On connaît l'importance reconnue aux substances corporelles en anthropologie de la parenté, et notamment aux « humeurs » (Héritier 1996) - les excreta-, par exemple au sang, à l'origine d'un répertoire symbolique très riche (Parkes 2001, 2004), ou encore au lait (Gélard 2005). Parmi ces substances, celle qui est aussi indissociable de l'être humain que la mort ne l'est de la vie est la chair. La chair peut être vive, corrompue, morte, ou encore morte et corrompue. C'est sur notre familiarité avec ce dernier aspect - l'état de décompositionque je vais maintenant insister. 


\section{Substance cadavérique et principe vital}

Tout au long de notre histoire évolutive, la présence de la corruption organique a-t-elle été suffisamment prégnante pour avoir des effets durables sur notre cognition? Probablement, au vu des données anthropologiques et historiques. Selon la thèse provocatrice de l'archéologue Lewis Binford (1988), nos ancêtres du paléolithique inférieur étaient davantage des charognards passif (accès tardif aux carcasses) que des chasseurs. Cette thèse a été fortement ébranlée, plusieurs travaux ayant démontré l'existence au paléolithique moyen de chasses pas seulement opportunistes mais aussi spécialisées (Speth 2012). Cependant, même si le charognage était marginal et essentiellement primaire (carcasses frâiches) sur le mode de ce que l'on observe de nos jours chez les Hadza (Joulian 1993), nos ancêtres du paléolithique et des époques plus récentes ont dû être régulièrement en présence de charognes animales, comme cela peut arriver encore aujourd'hui à toute personne s'aventurant dans des espaces non urbanisés. Jusqu'à nos jours, Homo sapiens lui-même, du fait de sa nature omnivore, a été régulièrement confronté à la corruption de nourriture, notamment animale, sauvage ou domestique. Par ailleurs, si comme l'a écrit Comte (1852: 153-154), notre « Grand-Être » se compose de beaucoup plus de morts que de vivants, il s'ensuit que les cadavres ont toujours fait partie de notre environnement, y compris sous leurs aspects les plus repoussants. Jusqu'au milieu du xix siècle, la pourriture nous a également été familière du fait de l'absence de traitement antiseptique des plaies accidentelles ou chirurgicales. Avant l'invention de l'antisepsie moderne par Joseph Lister, les conditions d'hygiène dans les hôpitaux étaient désastreuses et les infections, suite par exemple à des amputations de membres, allaient de 40 à 60 \% (Cohen 1999). Pyohémie, érysipèle et gangrène - le mot grec pour putréfaction - se répandaient facilement et lors de la guerre de 1870, en France, près de 80 \% des 13000 soldats qui subirent des amputations en moururent. Bref, qu'il s'agisse des charognes animales, de la corruption de nourriture, de la pourriture d'hôpital ou de la putréfaction des cadavres humains, la décomposition des corps a toujours accompagné l'histoire de notre espèce. Quels ont pu être les effets sur la pensée (nos représentations, nos croyances, le langage supposé les traduire) de ce fait massif?

Notons, en premier lieu, que la transformation et la disparition progressive de la substance (chair, sang, viscères, phanères, etc.) du cadavre est contre-intuitive à double titre. Elle l'est, d'abord, en ce sens qu'elle amène à penser qu'il y a autre chose dans la substance que ce qui se donne à voir, une puissance invisible et agissant sur elle. Elle l'est, ensuite, en regard du schème de la permanence que nous appliquons aux substances: intuitivement dotées d'une ontologie stable, elles ne devraient pas être significativement affectées par des déterminations circonstancielles. Dès lors, la violation de ce schème signifie que quelque chose se perd qui dénature la substance.

Par ailleurs, ce rapport à la pourriture a sans doute toujours été aussi violent perceptuellement - visuellement, olfactivement, tactilement - qu'il a été fréquent. On a donc tout lieu de penser que le traitement cognitif de cette violence a été lourd et coûteux, au point de marquer profondément notre esprit-cerveau et d'induire ou d'influencer des représentations d'un autre ordre. Un cadavre peut être chaud ou froid, souple ou rigide, sec ou humide, rester ferme ou se répandre, puer ou embaumer voire exhaler une « odeur de sainteté » (Albert 1990), mais son devenir naturel est de pourrir. De nombreuses données laissent penser que ce pourrissement a été perçu (et reste perçu) comme une perte de l'intégrité et de la pureté physique du corps de la personne défunte. Lors des rites funéraires, 
considérons par exemple le fait, bien documenté, d'un usage abondant de parfums, d'encens, de plantes odoriférantes, de fleurs, ces produits faisant fonction de contre-odeurs face aux émanations du cadavre. Sans occulter les aspects symboliques de ces pratiques, il semble bien qu'une de leurs finalités ait été de masquer le processus de corruption physique de la substance inhérent à la cadavérisation. Comment a-t-on pu passer ensuite de cette idée d'une impureté physique à des représentations plus abstraites du processus qui travaille la chair pourrissante?

« Dans l'affreux désarroi que nous éprouvons au spectacle d'une mort, écrit Mauriac (1934 : 26), il entre un sentiment de duperie: celui que nous aimons est là et il n'est plus là ».

Cette idée que la personne qui vient d'expirer n'est pas entièrement morte est l'objet de nombreuses illustrations ethnographiques dans cette mine de renseignements qu'est l'article "Contribution à une étude sur la représentation collective de la mort », publié par Robert Hertz en 1907 dans L’Année sociologique ${ }^{3}$. Dans beaucoup de sociétés, observe Hertz, il y a un délai d'attente qui peut aller jusqu'à plusieurs années entre la date de la mort et les obsèques définitives. Cette obligation s'explique parfois par les préparatifs longs et coûteux que nécessite la cérémonie finale, mais le plus souvent sa fonction est de laisser s'achever la décomposition du cadavre, jusqu'à ce « qu'il ne reste plus que des ossements ». Il est donc permis de penser, dit-il, « que normalement la période qui s'écoule entre la mort et la cérémonie finale correspond au temps jugé nécessaire pour que le cadavre passe à l'état de squelette » (Hertz 1928: 19), même si des causes secondaires peuvent prolonger ce délai.

Pourquoi cette attente du desséchement des os? Plusieurs explications sont avancées par Hertz.

« Si l'on tient tant à ce que la décomposition s'accomplisse, pour ainsi dire, en vase clos, dit-il, c'est qu'il ne faut pas que l'influence mauvaise qui réside dans le cadavre et qui fait corps avec les odeurs puisse se répandre au dehors et frapper les survivants » (ibid: 19).

Selon les Olo Ngadju, un groupe ethnique indonésien, un « nuage impur » environne le corps et souille tout ce qu'il atteint, les gens comme les choses. Les observations de l'anthropologue Céline Geffroy dans les Andes boliviennes (communication personnelle) font également songer à la même pollution olfactive. Selon les membres de la communauté paysanne où elle a mené sa recherche doctorale (Geffroy 2013), le cadavre, lors de la veillée mortuaire, dégage une odeur qui peut être très dangereuse pour les femmes enceintes ou les bébés alimentés au sein. Ces derniers sont susceptibles d'attraper la maladie du cadavre et plusieurs artifices sont mis en place pour déjouer ce risque qui s'atténue avec le temps. On peut relever une observation similaire dans la thèse d'Armelle Lorcy sur les Noirs et Indiens Chachi du littoral équatorien (2010) ou encore chez les Tanôsy de Madagascar qui jugent que le contact avec des morts abandonnés peut être à l'origine de tourments non seulement physiques mais aussi moraux car leur odeur funeste « abîme l'esprit » (Somda 2006 : 38).

Une autre explication, qui n'est pas sans lien avec la précédente, est l'impossibilité pour le défunt de rejoindre l'autre monde s'il n'est pas « sans chair », autrement dit, débarrassé de son impureté. Dans plusieurs îles mélanésiennes, ce n'est que lorsque l'odeur a disparu, dit-on, que l'âme cesse d'être un homme pour exister pleinement en sa qualité d'esprit protecteur.

«La réduction du cadavre à des ossements à peu près immuables, écrit Hertz, sur lesquels la mort n'aura plus de prise, apparaît comme la condition et le signe de la délivrance 


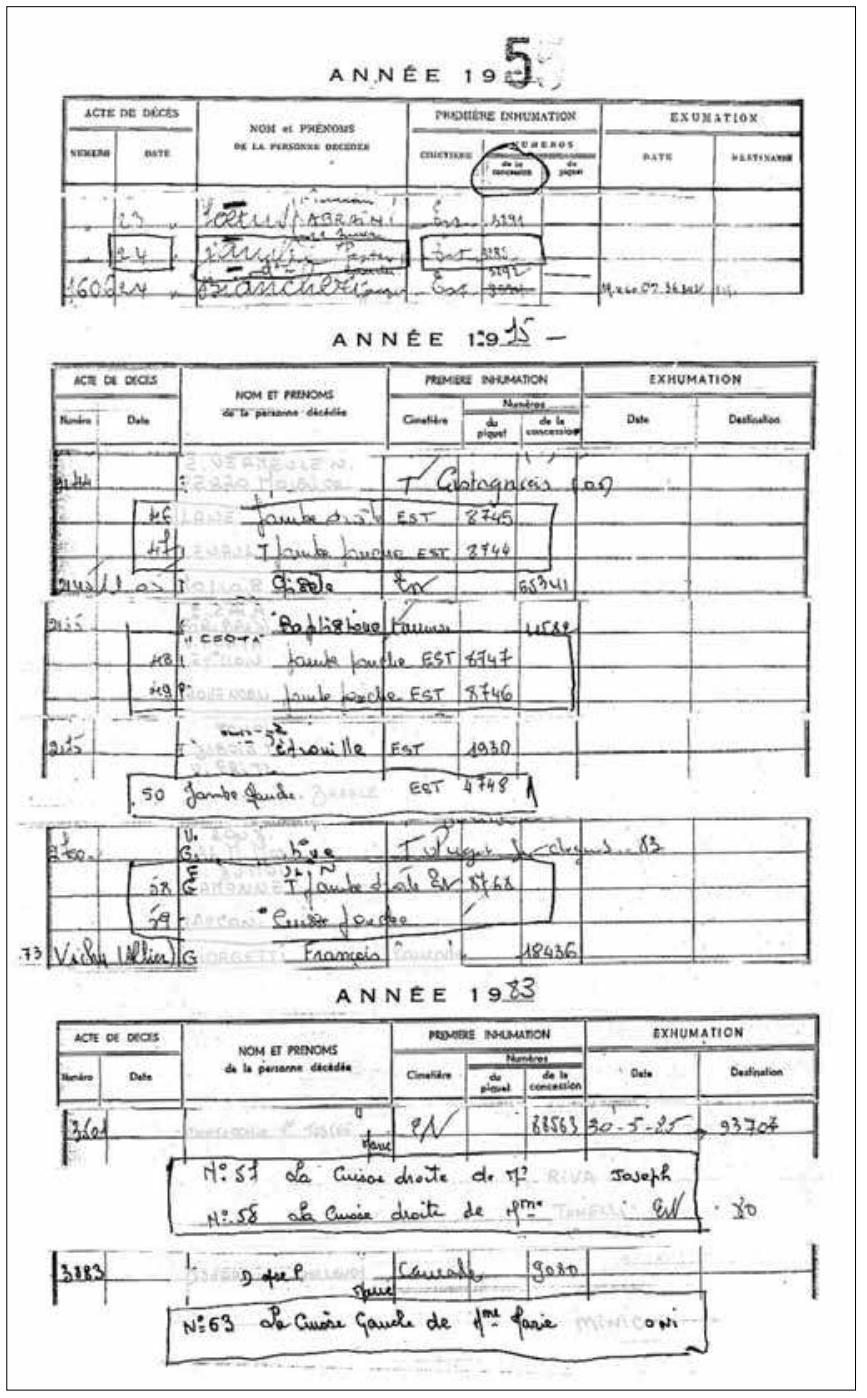

Extraits de registre des actes de décès

Mentions d'inhumations de membres amputés dans le registre des inhumations du service d'administration funéraire de la ville de Nice. je l'espère, mon propos. finale: maintenant que le corps du défunt est semblable à ceux des ancêtres, il semble qu'il n'y ait plus d'obstacle à l'entrée de l'âme dans leur communion » (Hertz 1928 : 75).

On retient de ces quelques exemples que, comme le disent les Ainu, un groupe ethnique de l'archipel nippon, « la mort n'est pas l'affaire d'un moment », elle n'est pas un acte instantané. Elle n'est pas représentée comme une destruction immédiate mais comme une transition. Bref, on ne meurt pas en un jour.

Si on ne meurt pas en un jour, il s'ensuit logiquement que le cadavre enferme encore un peu de la force vitale dont le corps est considéré comme un réceptacle dans de très nombreuses cultures (Lacaze 2000). Le processus graduel de la mort suppose que cette force vitale ou principe actif continue à œuvrer, raison pour laquelle les rites funéraires prévoient souvent l'offrande au corps du défunt de boissons ou de nourriture. Ce principe actif ne se perd donc pas immédiatement, il n'est pleinement consommé qu'au terme de la décomposition menée à son terme de la substance carnée. D'un point de vue cognitif, cette représentation a pu être renforcée par l'« effet Macbeth » qui, je le rappelle, associe impureté physique et impureté morale. L'impureté du corps corrompu devrait susciter une réprobation morale, mais ce n'est pas envisageable lorsque ce corps est celui de nos proches : parents, enfants, épouse ou époux, ami-e-s intimes. Il y a donc dilemme cognitif. La personne aimée, que l'on pleure, est en même temps objet d'une répugnance physique qui, dans ce cas précis, ne peut pas induire une répugnance morale. Mon hypothèse est que pour résoudre ce dilemme cognitif, nous en sommes arrivés à penser qu'il y avait autre chose dans ce corps que ce qui se donnait à voir sous une forme répugnante. Cette autre chose, c'est un principe caché qui est cause des effets observés (la pourriture, les odeurs) dans le moment même où il se détache du corps. Dans la partie suivante, je produis des données ethnographiques étayant,

\section{Pertes d'odeurs et dissipation du principe vital}

Considérons deux clauses du droit funéraire français. La première concerne ce que l'on peut assimiler à la mort d'une partie de nous-mêmes, l'amputation. Un membre amputé est la préfiguration ou l'objectivation partielle du cadavre que nous deviendrons un jour. Que faisons-nous de cette chose de chair et d'os, vouée à la décomposition 
et, à ce titre, fortement évocatrice de notre destin commun? Le décret n 97-1048 du 6 novembre 1997 relatif aux déchets d'activité de soins distingue, entre autres, les déchets anatomiques humains non identifiables (fragments d'organes ou de membres, kystes, fragments cutanés, placentas) et les pièces anatomiques d'origine humaine (organes ou membres issus d'amputations, fragments d'organes ou de membres, fragments cutanés, foetus). Les déchets anatomiques, supposés difficilement identifiables par un non spécialiste, sont éliminés comme des déchets d'activités de soins à risques infectieux. Les pièces anatomiques, en revanche, sont réputées être aisément reconnaissables par un non spécialiste. Elles doivent obligatoirement suivre une filière d'élimination spécifique (conditionnement, entreposage, transport, convention entre les professionnels) dont le terme est le traitement par un crématorium autorisé.

À mes yeux, le principe sur lequel se fonde ce texte est ce que Stéphane Ferret appelle un «symptôme d'identité » (1998: 16), c'est-à-dire « un indice permettant d'avoir de bonnes raisons de penser qu'il s'agit bien de cet objet ». Quel est cet objet dont on a de bonnes raisons de penser qu'il est ce qu'il est dans le cas des pièces anatomiques? Au-delà de l'identification possible d'un bras ou d'une jambe, l'objet ultime est la personne à qui appartient le membre amputé. Le bras ou la jambe est l'indice - au sens que ce mot a dans la trichotomie peircienne - d'un être humain et, à ce titre, le membre amputé est l'objet d'un traitement particulier, peu différent, en somme, de celui du corps du défunt. Cela est moins évident depuis le décret cité supra, supposé faire obligation (elle est controversée) d'incinérer les pièces anatomiques. Mais jusqu'à ce décret, les pièces anatomiques étaient souvent inhumées, comme j'ai pu le constater dans le registre des inhumations tenu depuis 1892 dans le service d'administration funéraire de la ville de Nice. On y compte une centaine d'inhumations de parties amputées du corps par $a^{4}{ }^{4}$, cela jusqu'en 1985, année à partir de laquelle toutes les pièces anatomiques sont « crématisées ». La quasi-totalité des mentions est au nom de la personne qui a subi une amputation d'un membre (jambe, bras) ou d'une partie d'un membre (cuisse, main, pied, genou).

Dans les extraits du registre reproduits à la page précédente, la mention, toujours très précise, indique le nom de la personne, parfois précédé de « M. » ou « Mme », souvent la latéralité du membre (cuisse droite, cuisse gauche) et le numéro de « piquet », fosse individuelle dans un terrain commun où le membre inhumé reposera cinq ans, avant d'être transféré dans l'ossuaire municipal. Exceptionnellement, il est fait mention d'un membre directement inhumé dans le tombeau familial (c'est le cas dans l'extrait du registre en date de 1955, où un numéro de concession est indiqué en regard de l'inhumation d'une jambe). La question reste posée de savoir si ces pratiques sont strictement locales - une enquête de grande ampleur sur le devenir des membres amputés dans diverses sociétés reste à faire -, mais elles confortent l'hypothèse selon laquelle le corps séparé - un membre ou une partie de membre -, à condition qu'il soit fait de chair et d'os, continue à être représenté comme conservant une part de la personnalité et du principe vital de la personne amputée. Dès lors, il peut être inhumé comme la personne le sera plus tard. Cependant, comme le corps tout entier, il perdra cette qualité au terme prévisible du processus de décomposition. En effet, après avoir reposé 5 ans au « piquet », le membre est transféré dans l'ossuaire municipal où la « raison sociale » des restes humains, si je puis dire, n'est plus identifiable.

La même logique semble être à l'origine d'une autre clause de la réglementation funéraire, relative à l'opération de réduction de corps, appelée aussi « recueillement ${ }^{5}$ ». « Lorsque le cercueil est trouvé en bon état de conservation au moment de l'exhumation, 
précise le Code général des collectivités territoriales, il ne peut être ouvert que s'il s'est écoulé cinq ans après le décès. Lorsque le cercueil est trouvé détérioré le corps est placé dans un autre cercueil ou dans une boîte à ossements ${ }^{6} »$.

L'objectif est de ne pas avoir à réduire un corps qui ne serait pas entièrement décomposé, car cet acte serait considéré comme une atteinte à l'intégrité du cadavre, notion qui a ici pour corollaire celle de personne. En revanche, le corps à l'état de squelette n'est plus soumis à ces restrictions. Dans ce cas, le corps séparé, qui n'est plus fait de chair et d'os mais d'os seulement, est sur le point de perdre sa personnalité, soit l'essentiel de son identité sociale ${ }^{7}$. Or ces os ne sentent plus, de l'avis même des fossoyeurs. Ils ne sont donc plus porteurs de puissances actives, contrairement au cadavre tant qu'il diffusait et perdait ses odeurs. Réduit à une substance osseuse, le cadavre perd alors son individualité - il n'a plus de "signature olfactive » (Dekeirsschieter et al. 2012) - et peut être mêlé à d'autres cadavres de la même tombe dans le cercueil de « recueillement ».

Un dernier argument me paraît conforter l'hypothèse d'une représentation du cadavre comme une substance travaillée par la perte d'un principe vital, processus qui se manifeste notamment par les odeurs qui en émanent. Lors de mes enquêtes sur les savoirs et savoir-faire olfactifs menées auprès de professionnels de la mort (médecins légistes, thanatopracteurs, fossoyeurs), les stimuli olfactifs propres aux cadavres étaient associés par mes informateurs à des termes dénotant tout à la fois la perte d'odeurs et de substance (Candau 2000, 2010b; Candau \& Jeanjean 2006). En voici quelques exemples:

« Il y avait donc cette liquéfaction hors destruction par les asticots, putréfactionliquéfaction de ce cadavre qui a dégouliné » (médecin légiste); « Alors ils avaient transporté le bonhomme là-dedans, et ça avait coulé... le type exsudait, exsudait, vous voyez » (médecin légiste); « L'odeur qui nous vient sur nous » (employé de la morgue); « Les odeurs restent pas au même endroit, elles s'éparpillent » (employé de la morgue); «Au niveau gastrique, ça dégage, quoi, ça dégage » (employé de la morgue); « Il y a une odeur qui se dégage » (employé des pompes funèbres); « L'odeur de charogne humaine, c'est vrai qu'elle est beaucoup plus..., elle s'imprègne, elle imprègne » (employé de la morgue); « Les personnes qui ont des escarres, j'ai toujours l'impression qu'elles sentent aussi de l'intérieur » (employé de la morgue); «Je ne sais pas, ce serait un ensemble,

Juanita, la momie des glaces

Dans la région d'Arequipa, momie découverte en 1995

dans la calotte glaciaire du Nevado Ampato,

à $6300 \mathrm{~m}$ d’altitude, parfaitement conservée.

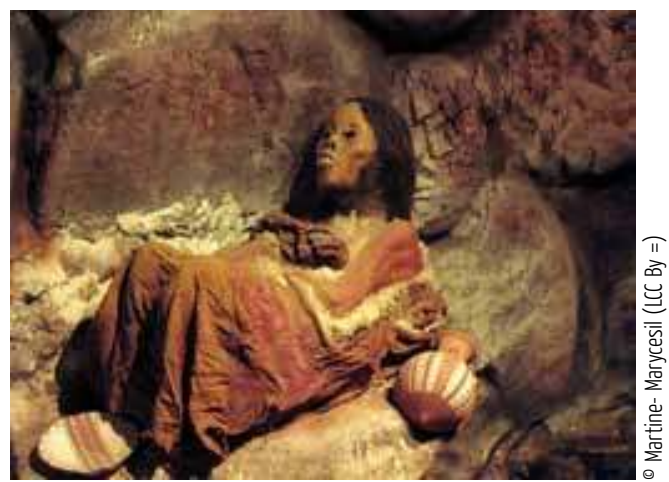


voilà, comme une odeur de..., de tran..., de décompo..., de rejet, heu..., comme de la transpira..., pas, on a l'impression que le corps est en train de faire un appel » (employé de la morgue); « C'est une habitude de prendre ces odeurs-là (fossoyeur), l'odeur, ça tombe dessus » (fossoyeur).

De la même manière que les corps se liquéfient, coulent, dégoulinent, suintent, fondent, exsudent, refoulent et sont assimilés, quand ils sont bien « avancés », à du «jus », de la « sauce », du « bouillon » (descripteurs utilisés par des fossoyeurs), les odeurs dégagent, diffusent, imprègnent, s'éparpillent, tombent et, par ce fait même, ont des effets: elles perforent, piquent, imprègnent, s'insinuent, s'incrustent. Les descripteurs olfactifs, pour la plupart, renvoient à des odeurs qui « viennent de l'intérieur » comme le dit explicitement un employé de la morgue. Ils sont, à mes yeux l'expression en langue, de représentations de l'être humain fortement pénétrées par les thèses du vitalisme que, bien après Aristote, on retrouve chez Paul-Joseph Barthez ${ }^{8}$, chez Marie François Xavier Bichat, puis chez Bergson ${ }^{9}$. En effet, tous connotent la tendance à l'épuisement progressif de l'énergie, des attributs et fonctions du corps ou, si l'on veut, une extinction de l'élan vital. Celle-ci achevée, le cadavre est dans un état où, toutes odeurs perdues, il ne sent plus: celui du corps desséché, c'est-à-dire, étymologiquement, le squelette ${ }^{10}$. Sa transsubstantiation est le fruit d'un processus de déperdition (des fluides, des masses, des odeurs) qui, arrivé à son terme, le retire définitivement du monde des vivants.

\section{$\&$}

Toute chose porte en elle la perte de sa substance, pourrais-je conclure en écho à la citation de James placée en exergue. Cette perte est intrinsèque au devenir du cadavre, mot dont l'étymologie même renvoie à la chute. Diverses pratiques sociales (momification, embaumement, cryogénisation) s'efforcent d'enrayer ce processus métamorphique total, mais elles ne font que le retarder, sans jamais pouvoir soustraire le cadavre au sort que lui promet la Genèse (3:19). J'ai soutenu dans cet article que nous étions enclins à associer cette perte de substance à la perte d'odeurs et à l'associer à l'épuisement d'un principe vital. « La vie est l'ensemble des fonctions qui résistent à la mort », écrit Bichat dans l'introduction des Recherches physiologiques sur la vie et la mort (Bichat 1822 : 2). De l'ensemble des données que j’ai livrées ici se dégage la représentation suivante, fondamentalement vitaliste: un cadavre qui pue, c'est encore de la vie qui résiste. Il n'est par conséquent pas surprenant que l'on ne puisse « réduire » un corps que lorsqu'il est à l'état de squelette et donc sans odeurs. Puer, je crois bien, est la seule et dernière forme d'expression sensorielle, mais aussi d'expression sociale, dont nous disposons une fois mort.

Je me risque à une ultime remarque, dont le caractère éminemment spéculatif ne m'échappe pas. Le cadavre est l'objet de nombreuses inférences cognitives et culturelles car, en sa présence, les personnes vivantes sont confrontées à des interrogations complexes. Comment continuer à chérir un proche quand son cadavre devient vermine? Comment réduire la dissonance cognitive et émotionnelle induite par l'attachement à une personne disparue et le caractère souvent repoussant de son corps pourrissant? Si 
la pureté du corps est associée à la pureté morale, comment rendre cette dernière qualité compatible avec l'impureté physique que représente la putréfaction? Une réponse intellectuellement satisfaisante à ces questions pourrait consister à dissocier le devenir ultime du corps et celui du principe vital, ce dernier ayant alors vocation à entrer dans le monde de l'au-delà et de la surnature. Des travaux anthropologiques donnent un certain crédit à l'hypothèse d'un enracinement de la pensée dualiste - au sens du dualisme des substances - dans le rapport que notre espèce a entretenu avec les cadavres. Pascal Boyer (2001 : 201-225) soutient que les propriétés des cadavres ont rendu certains concepts surnaturels pertinents et, à ce titre, ont contribué à l'émergence des croyances religieuses. Tout récemment, Marquet et al. (2012) ont repris cette hypothèse dans leur recherche sur les Chinchorro, un groupe de chasseurs-cueilleurs du désert d'Atacama (Chili) qui procédèrent à des momifications massives de cadavres entre 7000 et 4400 ans BP. Si cette hypothèse a quelque fondement, il faut alors envisager que la transsubstantiation du cadavre, dans sa puanteur la plus concrète, n'induit pas seulement la représentation d'un retrait progressif de la personne disparue du monde des vivants mais aussi celle de son accès à une autre forme de vie.

\section{NOTES}

Photo d'ouverture: De opwekking van Lazarus, école de Rembrandt Harmensz van Rijn, 1620-1650. @ Rijksmuseum Nederland. Fragment d'une représentation d'Oppien de Lazarus. À droite, la tombe ouverte de Lazarus est en partie visible. Des témoins divers apparaissent, incluant une femme à genoux, un homme en noir habillé de vêtements orientaux et un homme qui se protège contre la puanteur. [http://hdl.handle.net/10934/RM0001.COLLECT.5234].

1. http://furtherglory.wordpress.com/2010/11/04/whywere-jews-forced-to-scrub-the-sidewalks-of-vienna/ consulté le 25 novembre 2013.

2. Elles la conservent dans mon esprit-cerveau. Sur ce point, voir Sperber 1996 : 130 : « En ce qui concerne « charbon », personne ne soutiendrait que ce concept est inné, mais ce qui pourrait bien être inné, c'est la structure des concepts de substance, avec l'idée que les substances naturelles présentent des traits phénoménaux réguliers, en particulier la couleur ». Les substances s'offrent à nous dans un état naturel (l'eau, le lait, le cuivre), pré-artefactuel (le bronze, la viscose) ou synthétiques (certaines peintures), mais d'une manière générale on se les représente comme déjà-là - c'est indubitable dans leur état naturel - et à ce titre le rapport que nous entretenons avec elles semble bien plus ancré dans notre psychologie que celui des « choses » qui sont « séparables ».

3. Première série, tome $X,:$ 48-137.

4. En 1984, dernière année pour ce type de mentions, on en compte 96.

5. Le recueillement (ou réduction) des corps est la réunion des ossements des défunts appartenant à une même famille dans un seul cercueil ou le transfert des ossements d'une personne défunte dans un cercueil plus petit, généralement à la suite d'une détérioration du cercueil d'origine. Le recueillement est également pratiqué lors des exhumations administratives (concessions à l'état d'abandon ou non renouvelées).

6. Article R. 361-17 du Code des communes [art. R. 2213-42 du CGCT].

7. Cette perte ne concerne que le corps divisé des personnes ordinaires. Il en va différemment du corps des personnes extraordinaires, par exemple les rois ou les saints (songeons à l'importance des reliques), ou encore des corps «mémorialisés», tels ceux des victimes des génocides du Xx $x^{e}$ siècle.

8. Dans son introduction aux Nouveaux éléments de la science de l'homme, Barthez écrit (1778:1): «J'appelle principe vital de l'homme la cause qui produit tous les phénomènes de la vie dans le corps humain. Le nom de cette cause est assez indifférent et peut être pris à volonté. »

9. « Mais les causes vraies et profondes de division (du vivant) étaient celles que la vie portait en elle. Car la vie est tendance, et l'essence d'une tendance est de se développer en forme de gerbe, créant, par le seul fait de sa croissance, des directions divergentes entre lesquelles elle partagera son élan. » (Bergson 1959 : 66).

10. Skeletos vient de skellein « sécher (qqc)» et «se dessécher, se durcir, s'épuiser » (Robert historique). 


\section{POUR CITER CET ARTICLE}

Candau, J. 2013 Le Cadavre en subtance: perte d'odeurs et principe vital, in Guy, H.,Jeanjean, A. \& Richier, A., Le Cadavre en procès, TechniquesECulture 60 : 110-125.

\section{RÉFÉRENCES}

Albert, J.-P. 1990 Odeurs de sainteté. La mythologie chrétienne des aromates. Paris: Éditions de l'EHESS.

Barthez, P.-J. 1778 Nouveaux éléments de la science de l'homme. Montpellier: Imprimerie Jean Martel.

Bergson, H. 1959 L'Évolution créatrice. Paris: Presses universitaires de France.

Bichat, M. F. X. 1822 Recherches physiologiques sur la vie et la mort. Paris: Béchet Jeune et Gabon.

Binford, L. R., Mills, M. G. L. \& Stone, N. M. 1988 Hyena Scavenging Behavior and Its Implications for the Interpretation of Faunal Assemblages from FLK 22 (The Zinj Floor) at Olduvai Gorge, Journal of Anthropological Archaeology 7 (2): 99-135.

Boyer, P. 2001 Et l'homme créa les dieux. Comment expliquer la religion. Paris: Robert Laffont.

Candau, J. 2010b «Intersensorialité humaine et cognition sociale», Communications 86: 25-36.

- 2010a "Les formes partagées des affects olfactifs: des protoaffects aux affects représentationnels", Noesis 16: 129-154.

— 2001 « De la ténacité des souvenirs olfactifs». La Recherche 344: 58-62.

— 2000 Mémoire et expériences olfactives. Anthropologie d’un savoir-faire sensoriel. Paris: PUF.

- 1998 Mémoire et identité. Paris: PUF.

Candau, J., Jeanjean, A. 2006 « Des odeurs à ne pas regarder », Terrain 47: 51-68.

Carsten, J. 2011 Substance and Relationality: Blood in Contexts, Annual Review of Anthropology 40 (1): 19-35.

Chapman, H. A., et al. 2009 In Bad Taste: Evidence for the Oral Origins of Moral Disgust, Science 323: 1222-1226.

Cohen, J. 1999 Hospital gangrene: the scourge of surgeons in the past, Infection Control \& Hospital Epidemiology 20 (9): 638-640.

Comte, A. 1852 Catéchisme positiviste, ou Sommaire exposition de la religion universelle en onze entretiens systématiques entre une femme et un prêtre de l'Humanité. Paris: Chez l'auteur et Carilian-Goeury \& V. Dalmont.

Dekeirsschieter, J., et al. 2012 Enhanced Characterization of the Smell of Death by Comprehensive TwoDimensional Gas Chromatography-Time-of-Flight Mass Spectrometry (GCxGC-TOFMS), PLoS ONE 7 (6) : e39005. doi : 10.1371/journal.pone.0039005.

Ferret, S. 1998 L’identité, Paris: Flammarion.

Geffroy, C. 2013. Boire avec les morts et les pachamama. Une anthropologie de l'ivresse rituelle et festive dans les Andes boliviennes. Thèse d'anthropologie, Université de Nice Sophia Antipolis.

Gélard, M.-L. 2005 « La fourmi voleuse de lait. Transferts et représentations de la substance lactée dans le Tafilalt (Sud-Est marocain)», L'Homme 173: 97-118.

Hahn, H. P., Soentgen, J. 2010 "Acknowledging Substances: Looking at the Hidden Side of the Material World", Philosophy \& Technology, doi 10.1007/s13347-010-0001-8.

Haidt, J., Craig, J. 2008 The moral mind: How five sets of innate intuitions guide the development of many culture-specific virtues, and perhaps even modules. In P. Carruthers, S. Laurence \& S. Stich (eds.) The innate mind. New York: Oxford University Press: 367-392.

Haidt, J. 2001 The emotional dog and its rational tail: A social intuitionist approach to moral judgment, Psychological Review 108 (4): 114-134.

Héritier, F. 1996 Masculin, Féminin. La pensée de la différence. Paris: Odile Jacob. 
Hertz, R. 1928 Sociologie religieuse et folklore. Paris: PUF.

Holland, R. W., Hendriks, M. \& Aarts, H. 2005 Smells Like Clean Spirit. Nonconscious Effects of Scent on Cognition and Behavior, Psychological Science 16 (9): 689-693.

Jackson, D. D. 2011 Scents of Place: The Dysplacement of a First Nations Community in Canada, American Anthropologist 113 (4): 606-618.

Joulian, F. 1993. Chasse, "charognage" et hominisation, Préhistoire Anthropologie méditerranéennes 2: 7-14.

Kant, E. 1980 [1781 \& 1787] La Critique de la raison pure in Euvres philosophiques. Paris: Gallimard.

Kelly, D. 2011 Yuck!: The Nature and Moral Significance of Disgust. Cambridge, Mass.: MIT Press.

Lacaze, G. 2000 Représentations et techniques du corps chez les peuples mongols. Thèse d'ethnologie, Nanterre: Université Paris 10.

Liljenquist, K., Zhong, C.-B. \& Galinsky, A. D. 2010 The Smell of Virtue: Clean Scents Promote Reciprocity and Charity, Psychological Science 21 (3): 381-383.

Lorcy, A. 2010 Cuisiner les sensibilités. Alimentation, affects et société (Noirs et Indiens Chachi du littoral équatorien. Thèse d'Ethnologie et de Sociologie Comparative. Paris: Université Paris Ouest - Nanterre - La Défense.

Marquet, P. A., et al. 2012 Emergence of social complexity among coastal hunter-gatherers in the Atacama Desert of northern Chile, Proceedings of the National Academy of Sciences. 109(37) : 14754-14760.

Mauriac, F. 1934 Journal I, Paris: Grasset.

Moll J., et al. 2005 The moral affiliations of disgust, Cognitive and Behavioral Neurology 18: 68-78.

Park, J. H., Isherwood, E. 2011 Effects of Concerns About Pathogens on Conservatism and Anti-Fat Prejudice: Are They Mediated by Moral Intuitions? Journal of Social Psychology 151 (4): 391-394.

Parkes, P. 2001 Alternative Social Structures and Foster Relations in the Hindu Kush: Milk Kinship Allegiance in Former Mountain Kingdoms of Northern Pakistan, Comparative Studies in Society and History 43 (1) : 4-36.

Parkes, P. 2004 Fosterage, Kinship, and Legend: When Milk Was Thicker than Blood? Comparative Studies in Society and History 46 (3): 587-615.

Quine, W. V. 1977 Le Mot et la Chose. Paris: Flammarion.

Rozin, P., Haidt, J. \& Fincher, K. 2009 From Oral to Moral, Science 323: 1179 - 1180.

Schnall, S., et al. 2008a. Disgust as Embodied Moral Judgment, Personality and Social Psychology Bulletin 34(8): 1096-1109.

Schnall, S., Benton, J. \& Harvey, S. 2008 With a Clean Conscience: Cleanliness Reduces the Severity of Moral Judgments, Psychological Science 19 (12) : 1219-1222.

Shapiro, L. 2011 Embodied cognition. New York: Routledge.

Somda, D. 2006 Odeur des morts et esprit de famille (Anôsy, Madagascar), Terrain 47: 35-50.

Sperber, D. 1996 La contagion des idées. Paris: Odile Jacob.

Speth, J. 2012. Middle Palaeolithic subsistence in the Near East: zooarchaeological perspectives - past, present and future, Brain Farming 2: 1-45.

Van Leeuwen, F., et al. 2012 Regional variation in pathogen prevalence predicts endorsement of groupfocused moral concerns, Evolution and Human Behavior 33 (5): 429-437.

Varela, F., Thompson, E. \& Rosch, E. 1991 The Embodied Mind: Cognitive Science and Human Experience. Cambridge: MIT Press.

Zhong, C.-B., Liljenquist, K. 2006 Washing Away Your Sins: Threatened Morality and Physical Cleansing, Science 313: 1451-1452. 


\section{RÉSUMÉ}

Le cadavre en substance: perte d'odeurs et principe vital. Nous appelons « cadavre » la matière organique constitutive d'un individu décédé, telle qu'elle se donne à voir, à sentir ou à toucher, ou telle que nous l'imaginons quand le cadavre est absent ou disparu. En explorant les pratiques et représentations induites par la substance même du cadavre, cet article s'attache aux effets cognitifs et culturels de cette matière. Après avoir balisé théoriquement l'analyse dans la première partie du texte - théorie de la cognition incarnée et anthropologie des substances -, je rappelle dans la partie suivante que notre espèce a toujours entretenu une familiarité étroite avec le cadavre et sa transformation en charogne. Je soutiens ensuite que cette présence massive de la substance cadavérique dans la très longue durée a induit des représentations diverses, dont certaines très abstraites. Une de ces représentations est celle de principe vital, la perte ou la transformation de la substance du cadavre étant perçue comme la dissipation de ce principe. Dans la troisième et dernière partie, je défends l'idée d'un lien entre la décomposition du corps, la perte d'odeurs et la dissipation du principe vital, thèse que j'étaye par des données ethnographiques recueillies dans la région de Nice. Je conclus par quelques considérations relatives à la pensée dualiste.

\section{ABSTRACT}

The corpse in subtance: loss of smells and vital principle. We call «corpse » the essential organic matter of a dead individual, such as it shows, to smell or to touch, or such as we imagine it when the corpse is absent or disappeared. By exploring the practices and the representations inferred by the substance of the corpse, this article becomes attached to the cognitive and cultural effects of this material. Having marked out in theory the analysis in the first part of the text - theory of the embodied cognition and the anthropology of substances-, I remind in the following part that our species always maintained a narrow familiarity with the corpse and its processing in carrion. I support then that this massive presence of the deathly substance in the very long-lasting inferred diverse representations which some very abstract. One of these representations is the one of vital principle, the loss or the processing of the substance of the corpse being felt as the waste of this principle. In the third and last part, I defend the idea of a link between the decomposition of the body, the loss of smells and the waste of the vital principle, the thesis which I support by ethnographical data collected in the Nice's land. I concluded by some considerations relative to the dualistic thought.

\section{MOTS CLÉS}

cadavre, déchet, odeur, principe vital, substance, décomposition

\section{KEYWORDS}

corpse, waste, smell, vital principle, substance, decomposition 